\title{
Relevance of Aerodynamic Evaluation in Parkinsonian Dysarthria
}

\author{
Sarr Mamadou Moustapha1', Ghio Alain², Espesser Robert², \\ Teston Bernard ${ }^{2}$, Dramé Moustapha ${ }^{3}$ and Viallet François2,4 \\ ${ }^{1}$ UFR Santé- Université deThiès \\ ${ }^{2}$ Laboratoire Parole et Langage-Aix-en-Provence \\ ${ }^{3}$ Université de Reims \\ ${ }^{4}$ Service de Neurologie du Centre Hospitalier du Pays d'Aix-Aix-en-Provence \\ 1Sénégal \\ 2,3,4France
}

\section{Introduction}

Parkinsonian dysarthria is generally known under the name of hypokinetic dysarthria. Dysarthria, according to Darley et al (1969), is characterized by all speech disorders related to disturbances of muscular control of the speech organs, whose origin is a central or peripheral nervous system injury. So we must understand by dysarthria all failures related to either different levels of speech production (respiratory, phonatory, articulatory and even prosodic). Parkinsonian dysarthria, meanwhile, is mainly based on rigidity and hypokinesia. That's why it is considered as « hypokinetic » (Darley et al., 1975; Gentil et al., 1995). This term refers not only to reduction of articulatory movements but also to decreasing of speech prosody modulation described as monotonic (Viallet \& Teston, 2007). Parkinsonian dysarthria arises, like other signs of Parkinson's disease, the depletion of dopamine in charge of phonatory incompetence by muscular hypokinesia. It is a major handicap factor that may compromise in long-term oral communication of the patient, as worsening over the course of the disease, responding less well to treatment and thereby posing additional difficulties in support. So we thought to better assess this dysarthria in order to gain a better understanding and improve management. This assessment can be done by perceptual analysis. She could also be done by various instrumental methods (acoustic and physiological) focusing on one of the speech production levels mentioned above. Such studies are numerous in literature and we will report some examples in this chapter. What is more rare in literature is assessment of parkinsonian dysarthria in study combined several levels as might allow, for example, the dual approach appealing to physiology of speech production with firstly an aerodynamic component related to pneumophonic coordination (respiratory and phonatory levels) and, secondly, an acoustic component in relation to phonoarticulatory coordination (phonatory and articulatory levels). Through this chapter we want show that it is possible to assess appropriately parkinsonian dysarthria by using aerodynamic parameters that combine respiratory and phonatory levels, so such an experiment that we report in this chapter after having reviewed main methods of evaluation. 


\section{Perceptual analysis}

Perceptual analysis is subject to a large degree of subjectivity and inter or intra individual differences. However it can capture all functions involved in speech production system and is main foundation of parkinsonian dysarthria evaluation. On perceptual side, major disorder of parkinsonian speech is dysprosody. Prosody is defined as using of three vocal parameters (pitch, intensity and duration) which variations contribute to emotional and linguistic information. Parkinsonian voice is often described as low, monotonous, altered in timbre, too slow with hoarse character and difficult starting (Hartelius \& Svensson, 1994). In addition articulations'problems were reported including a certain loss of identity of phonemes, the most suitable example being realization of plosives $(/ \mathrm{t} /, / \mathrm{d} /)$ as fricatives (/ s /, / z /) due to insufficient closure of vocal tract (Robert \& Spezza, 2005). These disorders can occur very early during disease's course, perhaps as early as the clinical onset of it even at the presymptomatic stage (Harel et al., 2004). Dysphonia is first manifestation that appears early. It is secondarily complemented by articulatory disorders and airflow dysfunctions (Ho et al. 1998; Logeman et al., 1978). However articulatory disorders and airflow dysfunctions alter intelligibility more than dysphonia. Chronological order of disorders appearing suggests abnormalities progression down to up of the vocal tract during disease's course. Disorders begin at laryngeal level and end with bilabial constriction via lingual and palate constriction also. In all cases, perceptual marks of parkinsonian dysarthria were well reported by Selby (1968). Points of emphasis disappear, voice volume decreases, while consonants pronunciation is deteriorating and sentence ends in a whisper. At clinical onset of parkinsonian dysarthria, voice is low, monotonous (no variation in height). After, progressive worsening of dysarthria leads to inaudible and unintelligible diction. In some cases general slowness of movement is also reflected in speech rate. In others cases patients talk quickly, tangle words and sometimes carry words acceleration until sentence ending, imitating feast walking. Perceptual disturbances of Parkinsonian speech could also be summarized by identifying two clusters. On the one hand, a main cluster of prosodic insufficiency that combines monotony of pitch and intensity, accent reduction, quick acceleration, variable flow and consonants imprecision. On the other hand, an accessory cluster of phonatory incompetence that is related to voice disturbances.

Despite large amount of information it provides, perceptual analysis must be supplemented by more objective methods of assessment.

\section{Acoustic analysis}

Instrumental methods are generally limited in their analysis field. Despite this limitation, they allow, from quantified data, complex functions evaluation and objective comparisons between patients and normal subjects.

On acoustic side, perceptual impressions physical basis of Parkinsonian dysarthria have been studied by measuring several parameters.

\subsection{Fundamental frequency}

Measurements of voice fundamental frequency (F0) reported mixed results. However, most studies concluded that a F0 average increase in PD patients during sustained vowel, text reading or spontaneous speech (Flint et al. 1992; Hertrich \& Ackermann, 1993; Robert \& Spezza, 2005). For example, Ludlow and Bassich (1984) found a F0 average of $165.8 \mathrm{~Hz}$ for 
PD patients while F0 average value for control subject apparied in age and sex was $143.2 \mathrm{~Hz}$. As well Canter (1963) found F0 average values of $129 \mathrm{~Hz}$ for patients and $106 \mathrm{~Hz}$ for normal subjects. F0 increased with disease severity (Metter \& Hanson, 1986). Nevertheless, other studies have clearly demonstrated a F0 average reduction (Jankowski et al., 2004; Sanabria et al. 2001; Viallet et al., 2002,). It is therefore logical to agree on a certain diversity of trends in F0 that can be either lowered or increased or unchanged. F0 trends diversity could be due to biases related to patient age, gender, disease duration, variability of performance interand intra-individual as well as heterogeneity of measurement or evaluation methods. Regarding F0 variability in sentences production, it is reported much lower values in PD patients than normal subjects. Thus Canter (1963) noted frequency variations between 0.15 and 0.59 octaves for PD patients against 0.60 and 1.64 octaves for normal subjects. This limited variability observed in PD may be related to laryngeal rigidity that induces insufficient contraction including lack of crico-thyroid muscle which is mainly responsible for F0 increase. In sustained vowel task, there is disclosed an increase in F0 variability from cycle to cycle (Jitter) in patients, indicating an alteration of pneumophonic control stability (Jankowski et al, 2004).

\subsection{Intensity}

Regarding the vocal intensity, the results of perceptual analysis and acoustic measurements are not always consistent. For example, Fox and Ramig (1997) reported that the sonorous volume of PD patients was significantly lower than control subjects, around 2 to 4 decibels during speech production or other speech tasks such as sustained vowel. This result demonstrates clearly the hypophonic caracter of parkinsonian dysarthria. The results of other acoustic studies showed no significant differences between PD patients and normal subjects (Canter, 1963; Metter \& Hanson, 1986). The alteration or no of the sonorous volume rather depend on the degree of severity of illness (Ludlow \& Bassich, 1984). Despite these mixed results, however, there would be leaning towards a small reduction of mean intensity which falls within the phonatory incompetence associated with the subglottic pressure decreasing. The shimmer is for intensity what the jitter is for frequency, and it reflects intensity variability of sound vibration from cycle to cycle. A shimmer increasing in the task of sustained vowel has been reported in Parkinson's disease (PD) patients compared with control subjects, indicating an alteration of laryngeal stability control (Jimenez et al., 1997). These findings suggest that a reflex part of speech production control appears to be intact, contrary to the dysfunction of voluntary control directly induced by the disease.

\subsection{Abnormalities of vocal timbre}

Acoustic measurements during sustained vowel confirmed the perceptual abnormalities of timbre (blown, frayed or tremulous character) in addition to showing F0 and intensity increasing variability from cycle to cycle, changes longer term due mainly to the tremor, with a reduction of signal/noise ratio (Viallet \& Teston, 2007).

\subsection{Speed of speech}

The speed of speech of PD patients is highly variable from one subject to another (Darley et al, 1975). Some studies showed no significant difference between parkinsonian and normal subjects (Ackermann \& Ziegler, 1991; Ludlow et al. 1987). Other studies have reported a 
faster speech rate in PD patients (Weismes, 1984). Finally, the speech speed can also be slower (Volkmann et al., 1992). These differences reflect not only the variability between subjects, but also the possible variation of results depending on the task (Ho et al., 1998). In all cases, Parkinsonian speech is marked by abnormalities which are described a long time and may impact on speed: festination, palilalia and pseudo-stuttering with dysfluences (Monfrais-Pfauwadel, 2005). What is more, the fine analysis of the acoustic signal from read speech extracts with attentive listening has led to a better study of the Parkinsonian speech temporal organization: the speed of speech tends to be slower. This slowness seems correlated with a longer pause time, duration of breaks was found significantly higher in PD patients compared with control subjects (Duez, 2005). In addition breaks inside of words have been observed in PD patients and not in controls subjects. Finally many dysfluences, such as omissions, repetitions and false starts, were found almost exclusively in PD patients. Numerous breaks and dysfluences not only slow the speed of speech, but also deconstruct the language units, disrupt perceptive waiting of listeners and finally degrade intelligibility.

\subsection{Imprecise consonants}

The most typical perceptual error articulatory in Parkinsonian dysarthria, namely the realization of consonants as fricatives was also confirmed by the acoustic analysis. In effect during these tests, it is found, instead of a silence due to normally carried out occlusion, a signal corresponding to a low intensity friction noise due to air passage and defined as the spirantisation phenomenon. Similarly, the lack of acoustic contrasts reflecting a lack of articulation is a common feature of parkinsonian speech spectrograms (Kent \& Rosenbek, 1982).

\subsection{Other anomalies}

Finally, other deviations were reported always in acoustic studies: the reduced duration of formant transitions (Connor et al. 1989; Forrest et al., 1989), the voicing of voiceless consonants assigned to the rigidity of the larynx, a control loss of voice onset time (VOT), that is to say, the time between the release of the consonant and the beginning of voicing, resulting in a lack of coordination between the larynx and articulatory organs (Forrest et al., 1989; Lieberman et al., 1992).

\section{Physiological analysis}

It essentially uses electromyographic methods, vidéocinematographic, kinematic and aerodynamic. It provides quantitative data on respiratory plans, phonatory and articulatory (Teston, 2007).

\subsection{Respiratory system}

Kinematic studies have measured the thoracic and abdominal movements. The spirometric measurements allowed to assess the volumes of mobilized air during inspiration and expiration. At rest PD patients respiration is characterized by a shortening of respiratory cycle at the expense of expiration and, moreover, a relative decline of thoracic participation in respiratory movement. During speech production, it was noted in PD patients an inspiratory volume reduction of the thoracic cage, and an increase in inspiratory abdominal volume, which suggests an alteration of expiratory airflow necessary to set the appropriate contribution of laryngeal vibrator (Solomon \& Hixon, 1993). 


\subsection{Phonatory system}

The rigidity of the laryngeal musculature is a major determinant of hypophonia associated with parkinsonian dysarthria. It has been demonstrated by studies in laryngoscopy which provided direct light on the anomalies of the larynx. Larynx anomalies include glottal gap by chord adherence default, sometimes hypertonia of ventricular bands and tremor which can be localized at chordal level or above glottal part of vocal tract (Jiang et al., 1999; Yuceturk et al., 2002). Laryngeal rigidity induces a particularly curved form of vocal cords responsible for the unusually large and constant aperture of the vocal tract (Smith et al., 1995).

\subsection{Articulatory system}

On physiological side it is mainly explored by electromyographic and kinematic methods. In fact electromyographic and kinematic methods permit to analyze strenght and movement of articulatory organs in order to better understand the motor speech disorders

\subsubsection{Articulatory organs movement}

The mobility of articulatory organs of speech, like other movements, is disturbed by two major symptoms of Parkinson's disease: rigidity and hypokinesia.

The rigidity incrimination has been strengthened on the basis of certain works. For example Hunker et al. (1982) were able to evaluate a coefficient of rigidity by applying known forces on labial muscles and observing the resulting displacement. The lower lip of PD patients showed a significantly higher rigidity than control subjects, whereas for the upper lip, there was no significant difference between the two groups. Moreover a correlation between the degree of rigidity and the movements' reduction was observed by recording the lips movement with a strain- gauge system in connection with the muscular activities of inferior orbicularis and mentalis, (Barlow et al., 1983). However, this rigidity is not expressed identically on all articulatory organs, affecting preferentially muscles which are poor in neuro-muscular spindles and without stretch reflex such as the tongue comparatively to other muscles which are richer in neuro-muscular spindles and with monosynaptic reflex activity such as the jaw elevators (Abbs et al., 1987).

The hypokinetic character of some articulatory movements during parkinsonian speech is reported in particular by Ackermann et al. (1993). In this study recording the lips and tongue movements with an electromagnetic system during the repetition of syllables [pa] and [ta], there was an increased frequency and decreased amplitude of articulatory movements during the repetition of the syllable [ta] and no anomaly was found during the repetition of the syllable [pa]. This result suggests that there may be different mechanical properties between the tongue and lips. Kinematic studies also showed that hypokinesia of muscles, thus the nature of motor performance, may depend on factors such as familiarity of the task, the existence of visual guidance (Connor and Abbs, 1991) or even speed of speech (Caligiuri, 1989). Finally the kinematic studies have also confirmed, in PD patients, lack of coordination between different muscles involved in the complex activity that is speech production. Indeed the kinematic analysis of jaw, upper and lower lip showed a different motor behavior of these three structures. The lower lip was working normally when the upper lip and jaw had velocity peaks and/or reduced amplitude of movement (Connor et al., 1989). 


\subsubsection{The articulatory organs forces}

It is usually assessed by using force transducers (Barlow et al., 1983). Muscle abnormalities are also detectable by using electromyographic explorations (Leanderson et al., 1971). The latter, despite their relative inaccessibility to non-medical researchers and the difficulties attached to their technical realization and interpretation, can provide a wealth of information on the chronology of muscular events and agonist-antagonists relation (Teston, 2007). It has been noted in parkinsonian dysarthria abnormal electromyographic signal during the study of orbicularis upper lip activity in repetition of the syllable [pa]. Indeed, in PD patients comparatively with control subjects and during repetition, the short bursts of muscle activity associated with each syllable had duration of shorter and shorter with an associated reduction in their amplitude (Netsel et al., 1975).

These physiological analysis concerning only one level of peripheral production of speech should be more and more replaced by the combined study of at least two levels; example of such a combined analysis is provided by the study of pneumophonic coordination.

\subsection{The pneumophonic coordination}

It reflects the synergy of action that must exist during speech production between respiratory and laryngeal levels. The measurement of subglottic pressure (SGP) is a good indicator of this pneumophonic coordination. Indeed, the SGP is evaluable indirectly via the intraoral pressure (IOP) during the production of plosives and depends on both the expiratory airflow and laryngeal resistance. In other words, SGP results from a dynamic conflict between air thrust forces and laryngeal resistance, so the evaluation of its trend in a group of breath can give a powerful index of the speaker pneumophonic coordination (Teston, 2007). So such a parameter, related to the aerodynamic side of speech production with in addition its non-invasive character, can be relevant in the assessment of parkinsonian dysarthria.

\section{Relevance of the evaluation of aerodynamic parameters}

Our research team has experience of using aerodynamic parameters in the assessment of parkinsonian dysarthria. The measurement of such parameters has been performed in PD patients and control subjects by using the voice evaluation system Eva 2 of SQ LAB society in Aix-En-Provence.

\subsection{Used parameters}

We worked primarily on three parameters: the intra-oral pressure (IOP), the mean oral airflow (MOAF) and laryngeal resistance (LR).

IOP is an indirect reflection of subglottic pressure which is itself nothing other than the pressure exerted by the expiratory air column on the vocal cords. Subglottic pressure is an important aerodynamic parameter and could allow a better understanding of some dysfunctions in speech production system (Baken \& Orlikoff, 2000).

The MOAF is another important aerodynamic parameter associated with the laryngeal function and speech production. MOAF and subglottic pressure allow together a better description of the aerodynamic component of speech production.

Finally, the LR is the ratio of IOP on the MOAF and should be able to give an idea about the functioning level of laryngeal stage. 


\subsection{Equipement and measurement technique}

\subsubsection{Equipement}

We used in this study the vocal evaluation system EVA 2 developed by the Laboratory of Speech and Language and sold by SQ-Lab society. EVA 2 operates as a workstation PC in the Windows environment (See Figures 1 and 2) with different software applications dedicated to acoustic and aerodynamic analysis of speech production.

The recording device includes an acoustic channel and two aerodynamic channels: one for measurement of mean oral airflow (MOAF), the other for the IOP measurement. It is thus possible to measure IOP during holding of a voiceless plosive. As a reminder, IOP is the estimated subglottic pressure.

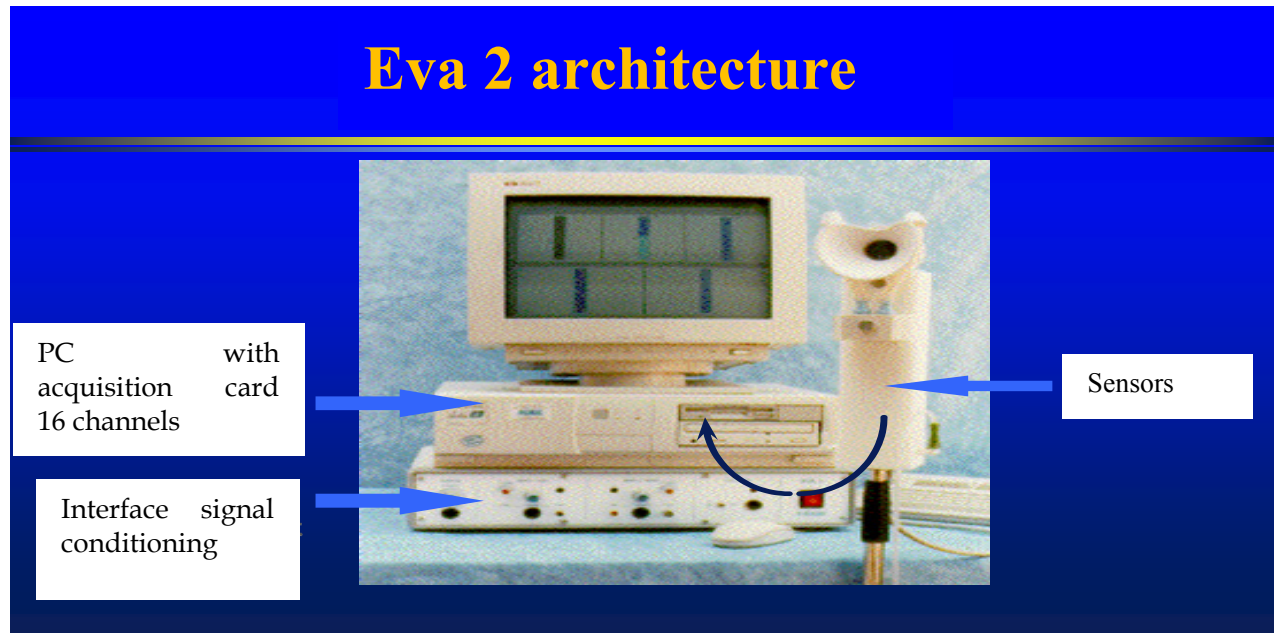

Fig. 1. General Feature of Eva 2 (workstation PC in the Windows environment)

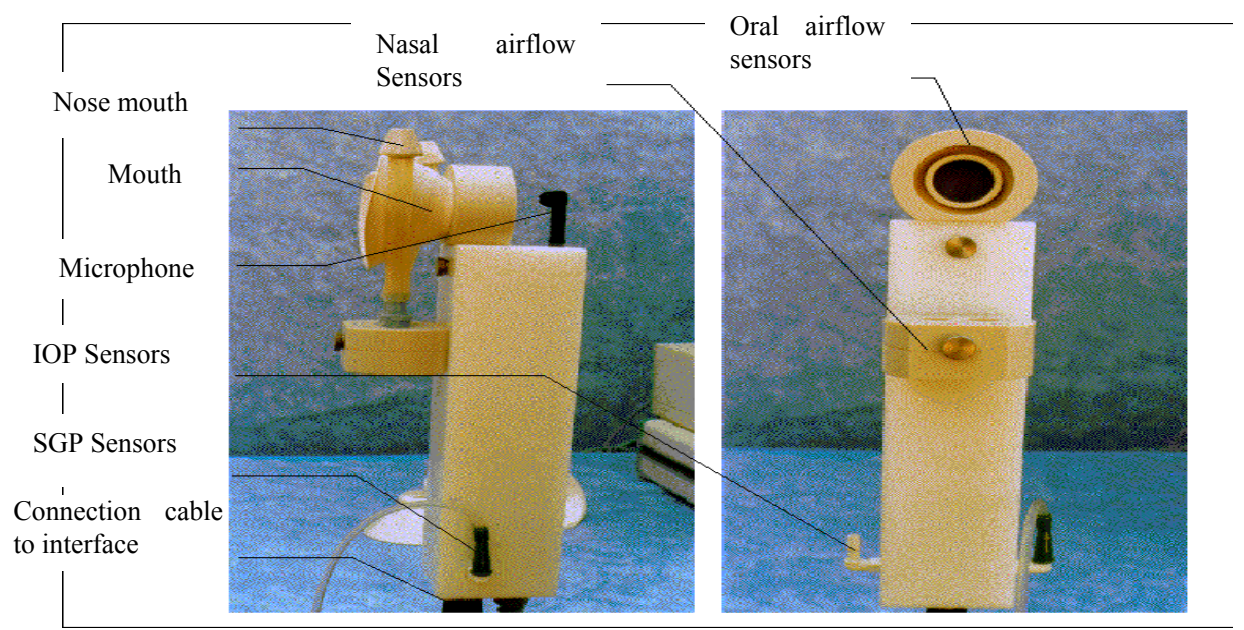

Fig. 2. EVA 2 hand piece with accessories (microphone, mouth, sensors etc.) 


\subsubsection{Technique}

The measurement technique derives from the general theory of fluids dynamic applied to the airway. According to this theory it is possible by adjustments of valves to estimate pressure-flow upstream from the direct measurement of pressure-flow downstream of the target site. The adjustments of valves in question occur naturally during the pronunciation of certain sounds. For example, during production of the consonant / p / the lips are closed while the glottis is open. In contrary during pronunciation of the vowel / a / the lips are open while the glottis is closed. The different conformation of these examples of valves located on the airway (glottis and lips) has a physical impact on the pressure and flow dynamics prevailing inside airway. So during the realization of a voiceless plosive (/ p /), there is a momentary equilibration of intra-oral and subglottic pressures. This equilibration allows indirect assessment of SGP (upstream) via the direct measurement of IOP (downstream). The momentary equilibration of subglottic and intra-oral pressures occurs when holding the voiceless plosive because at this moment there is no phonation, the lips are closed and the glottis is open. Thus the peak pressure generated by holding a voiceless plosive may be considered as a "snapshot" of the subglottic pressure immediately preceding phonation. Similarly during the realization of the vowel (/ a /) following a voiceless plosive (lips are open and glottis is closed), it is possible to consider the oral airflow as a snapshot of translaryngeal airflow because of continuity of flow through the upper airway when the mouth is open. Once we got the two parameters, it suffices to calculate the ratio of intra-oral pressure on the oral airflow to determine the laryngeal resistance (Smitheran \& Hixon, 1981; Demolin et al, 1997) (See Figure 3).

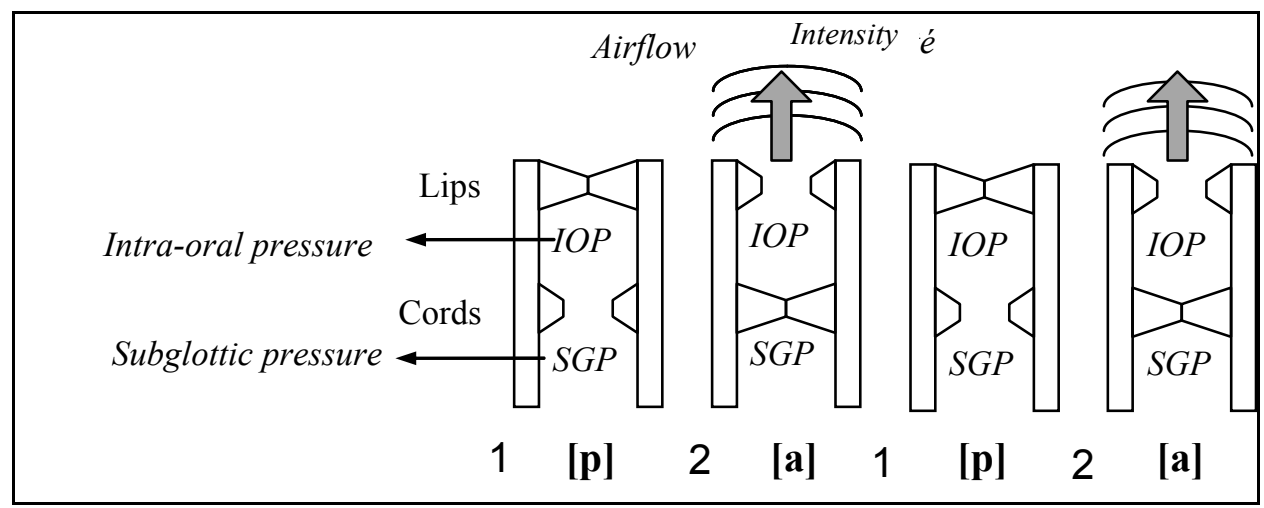

Fig. 3. Evaluation of the subglottic pressure.

Intraoral pressure (IOP) is equivalent to the subglottic pressure (PSG) during the labial occlusion of phoneme "p". Subglottic pressure is estimated indirectly by "Interrupted Airway Method" (Smitheran \& Hixon, 1981), method validated notably by Demolin et al. (1997).

Measurements were performed while the subject produced at a constant rate the sentence "Papa ne m'a pas parlé de beau-papa" (Daddy did not speak to me about daddy-in-law). During this production, oral mouth was firmly against the underside of the face to minimize air leakage (see Figure 4). Taking IOP is performed using a disposable suction catheter approximately $4 \mathrm{~mm}$ (See Figure 5). The probe was placed between the incisors and should not be crushed between the teeth or be obstructed by saliva. 


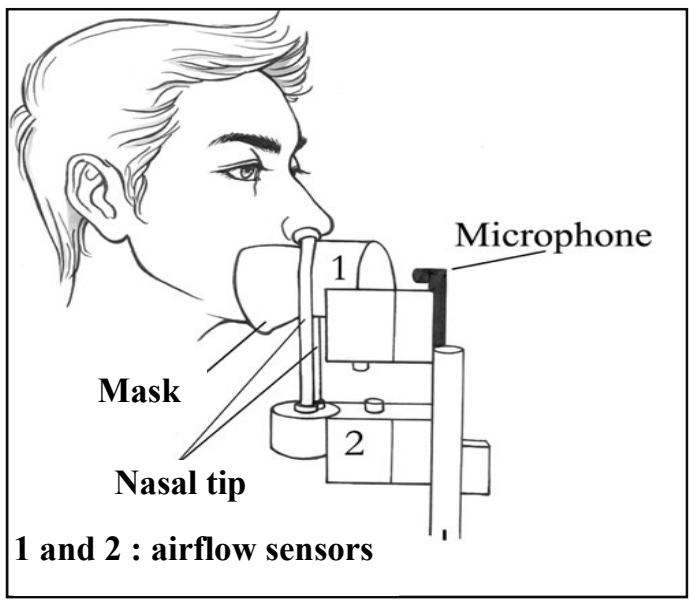

Fig. 4. Oral mouth firmly against the underside of the face

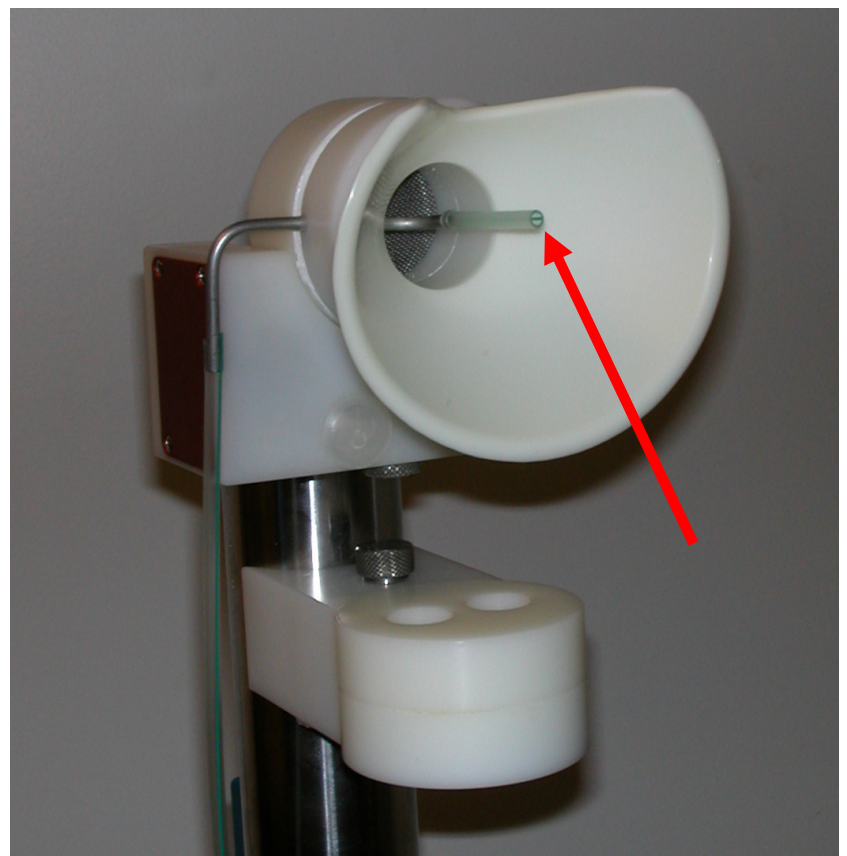

Fig. 5. Note the suction probe for taking IOP (indicated by red arrow)

\subsection{Patients and control subjects}

The study included 24 subjects with PD who had an average age of 59 years (SD $=5.65)$ with a mean duration of disease about 9,9 years $(\mathrm{SD}=3.27)$. Patients were recorded after withdrawal of L-dopa for at least $12 \mathrm{~h}$ (condition called OFF DOPA). 
50 healthy subjects served as controls. They had an average age of 61 years $(\mathrm{SD}=10,5)$.

\subsection{Statistical analysis}

Statistical comparisons between groups (CTRL vs. OFF DOPA) were conducted on the basis of a linear mixed model (software "R" version 2.6.2, http://www.r-project.org). This model emerged as best suited to the analysis of grouped data. Indeed, the repeated measurements, longitudinal studies are data that are presenting a group structure. And in our case, a single individual is undergoing multiple measures, and structured data in this way no longer meet one of the fundamental prerequisites for the validity of a classical linear model, namely the independence of measures. We set our statistical comparisons as follows: measurements of aerodynamic parameters (IOP, MOAF and LR) accounted for the numerical factor of the model, the group (CTRL, OFF DOPA), the position of the consonant / p / in the sentence produced (P1, P2, P3, P4, P5, P6) and the subject (patients, controls) were the three factors model variability.

A p-value less than 0.05 was accepted as statistical significance.

\subsection{Results}

In a study that involved 20 male patients registered in terms ON / OFF STIM and 11 control subjects, measurement of IOP showed a statistically significant fall of this parameter in OFF STIM patients compared to controls. The stimulation of Subthalamic nucleus (STN) improved partially IOP with a statistically significant difference at the first two measurement points whereas there was an effect of convergence on the third point (Sarr et al., 2009).

In another study that focused on 24 patients registered in OFF DOPA condition and compared with 50 control subjects, three parameters (IOP, MOAF and LR) were measured on six / P / (P1 to P6) of the sentence « Papa ne m'a pas parlé de beau papa » that subjects pronounced at a constant rate.

Here too, there was, as regards the IOP, a statistically significant decrease in patients compared to controls $(p=0.0001)$ (See Table 1 and Figure 6).

\begin{tabular}{|l|c|c|c|c|c|c|}
\hline & P1 & P2 & P3 & P4 & P5 & P6 \\
\hline \multirow{2}{*}{ OFF DOPA } & 3,84 & 6,22 & 4,46 & 4,7 & 4,49 & 4,26 \\
& $(1,9)$ & $(2,2)$ & $(1,8)$ & $(1,9)$ & $(1,9)$ & $(1,7)$ \\
\hline \multirow{2}{*}{ CTRL } & 5,23 & 6,97 & 5,73 & 5,9 & 6,06 & 5,67 \\
& $(2,00)$ & $(2,15)$ & $(1,90)$ & $(1,93)$ & $(1,98)$ & $(2,00)$ \\
\hline
\end{tabular}

Table 1. Average of intraoral pressure (IOP) in control subjects (CTRL) and OFF DOPA patients at six measurement points. Standard deviations are in parentheses.

Concerning mean oral airflow (MOAF) the curve of mean values at six points of measurement in control subjects (CTRL) and OFF DOPA patients showed an convergent aspect at extremities so that P1 and P6 while at the other measurement points (P2 to P5), the two curves were clearly separated: that of control subjects remain above that of OFF DOPA patients (see Table 2 and Figure 7). The comparison between the two groups was statistically significant $(\mathrm{p}=0.001)$. 


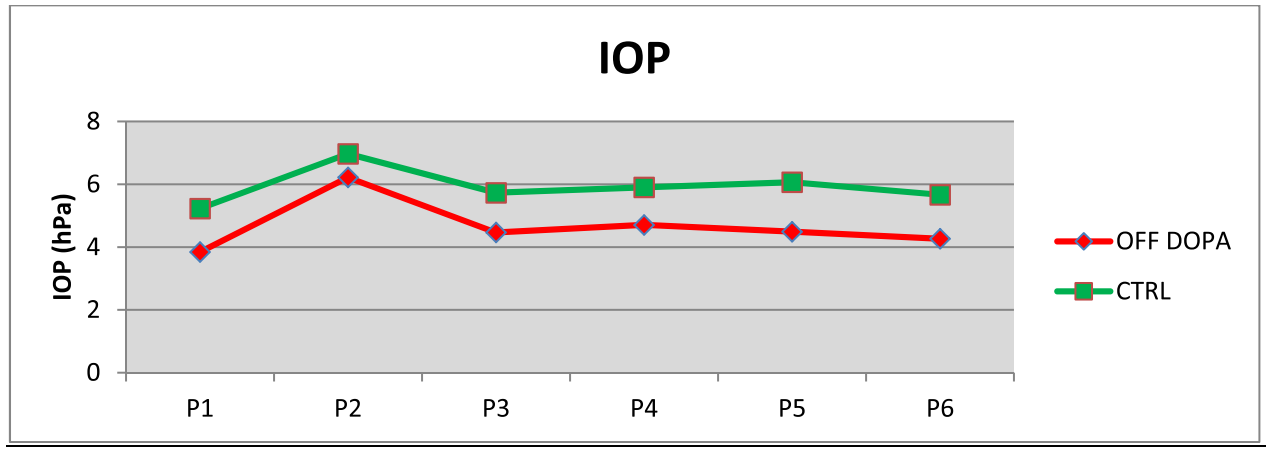

Fig. 6. Curve of the intra-oral pressure (IOP) in control subjects (CTRL) and OFF DOPA patients at six measurement points.

\begin{tabular}{|l|c|c|c|c|c|c|}
\hline & DAOM 1 & DAOM 2 & DAOM 3 & DAOM 4 & DAOM 5 & DAOM 6 \\
\hline OFF & 0,2 & 0,16 & 0,17 & 0,17 & 0,19 & 0,2 \\
DOPA & $(0,09)$ & $(0,08)$ & $(0,08)$ & $(0,08)$ & $(0,07)$ & $(0,08)$ \\
\hline \multirow{2}{*}{ CTRL } & 0,2 & 0,21 & 0,21 & 0,20 & 0,21 & 0,2 \\
& $(0,08)$ & $(0,07)$ & $(0,07)$ & $(0,08)$ & $(0,06)$ & $(0,06)$ \\
\hline
\end{tabular}

Table 2. Average of mean oral airflow (MOAF) in control subjects (CTRL) and OFF DOPA patients at six measurement points. Standard deviations are in parentheses.

NB: DAOM is the french abbreviation of mean oral air flow (MOAF)

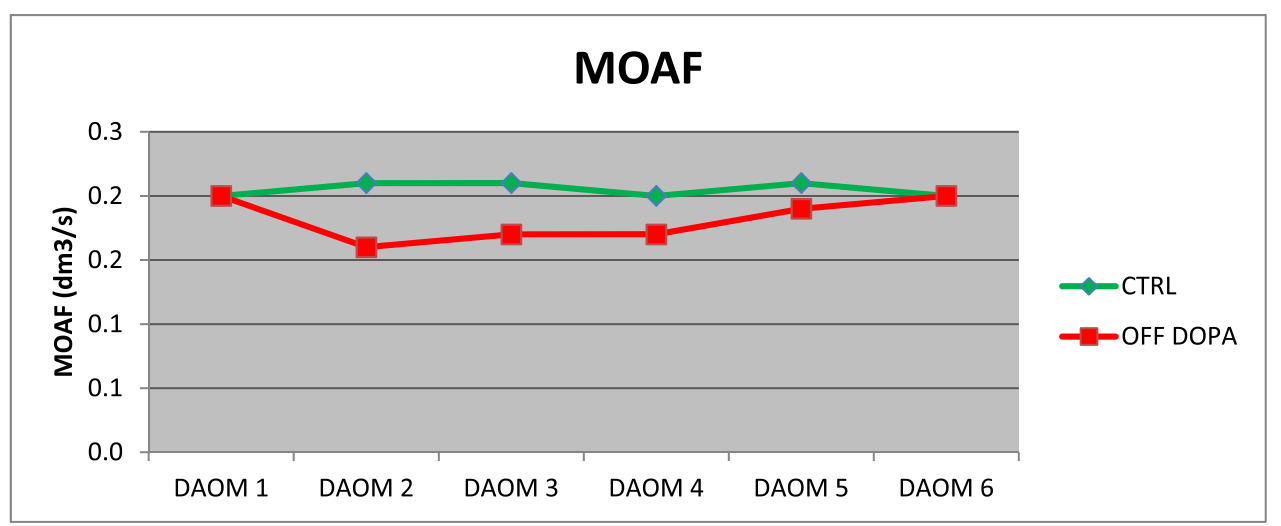

Fig. 7. Curve of mean oral airflow (MOAF) in control subjects (CTRL) and OFF DOPA patients at six measurement points.

Finally for the LR, the graphical representation of mean values at six points of measurement in control subjects (CTRL) and OFF DOPA patients showed on one hand a more linear overall appearance of the control-subjects 'curve, on the other hand, a curve of OFF Dopa patients above that of control subjects from P1 to P4 and then, below, beyond P4. In addition standard deviations were significantly larger in OFF DOPA patients than in control subjects (See Table 3 and Figure 8). The comparison between the two groups was statistically significant $(p<0.05)$. 


\begin{tabular}{|l|c|c|c|c|c|c|}
\hline & RL 1 & RL 2 & RL 3 & RL 4 & RL 5 & RL 6 \\
\hline OFF & 28,05 & 51,22 & 33,77 & 33,99 & 27,09 & 29,21 \\
DOPA & $(20,50)$ & $(40,14)$ & $(23,1)$ & $(21,97)$ & $(16,27)$ & $(26,74)$ \\
\hline \multirow{2}{*}{ CTRL } & 25,75 & 35,38 & 30,81 & 33,40 & 30,84 & 33,64 \\
& $(16,92)$ & $(13,54)$ & $(11,83)$ & $(14,09)$ & $(11,64)$ & $(13,51)$ \\
\hline
\end{tabular}

Table 3. Mean of laryngeal resistance in control subjects (CTRL) and OFF DOPA patients at six measurement points. Standard deviations are in parentheses.

NB: RL is the french abbreviation of laryngeal resistance (LR)

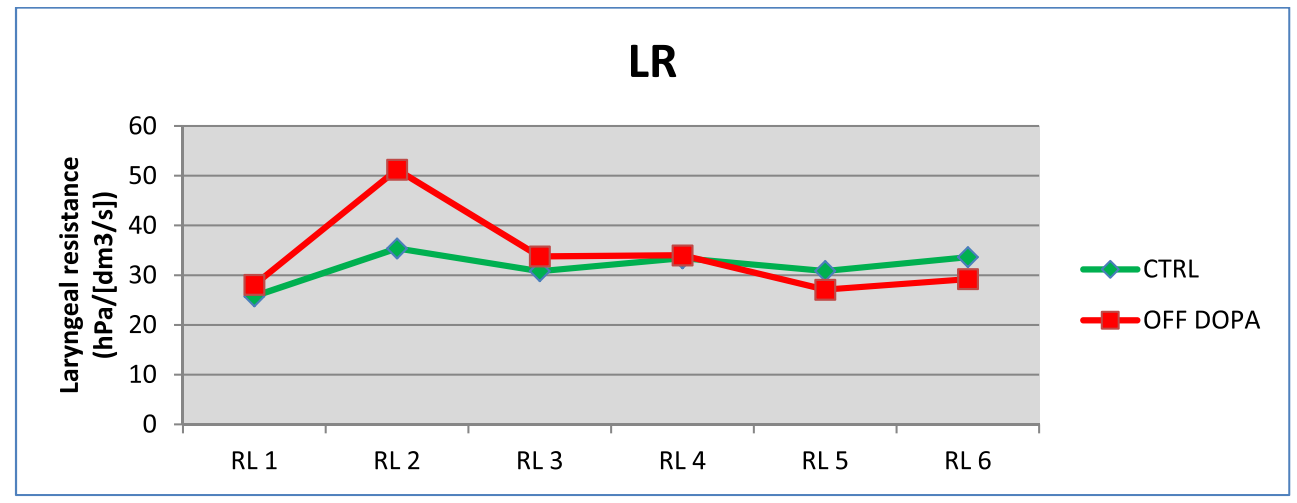

Fig. 8. Curve of mean values of laryngeal resistance (LR) in control subjects (CTRL) and OFF DOPA patients at six measurement points.

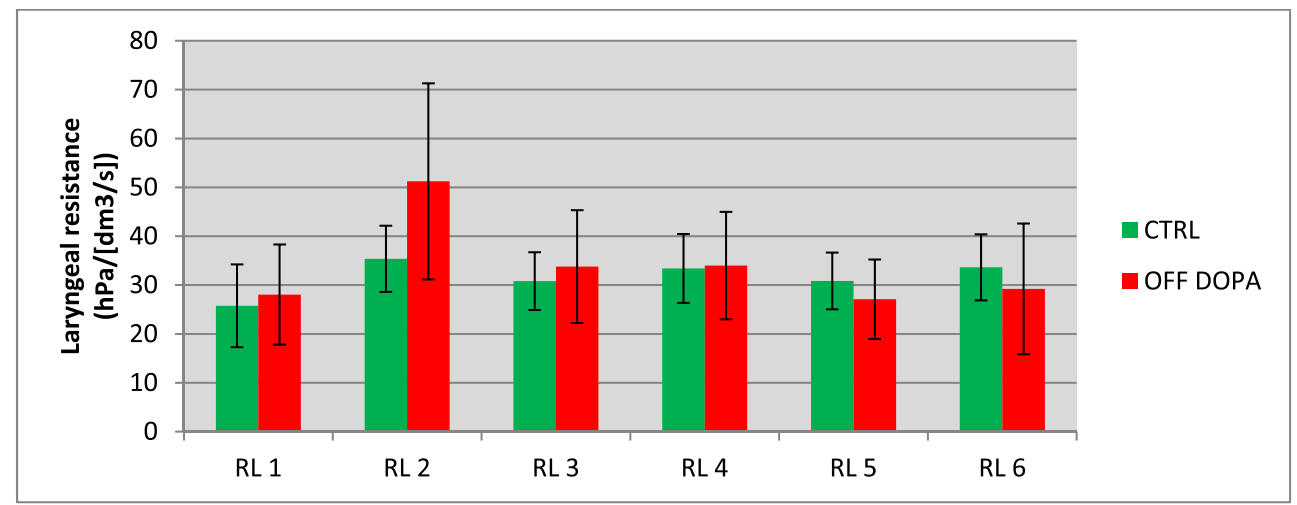

Fig. 9. Histogram of mean values of laryngeal resistance (LR) in control subjects (CTRL) and patients OFF DOPA at six measurement points, with standard deviations. The histogram allows to better see the standard deviations significantly larger in patients.

\subsection{Discussions}

This new study that examined 24 patients and 50 control subjects confirms the decrease of IOP on all six measurement points of the sentence when comparing patients with control 
subjects. The decrease of IOP, found in a previous study (Sarr et al., 2009), seems to confirm definitively the alteration of this parameter in parkinsonian dysarthria. The decrease of IOP in patients is due to dopamine deficiency inherent in PD. Dopamine deficiency induces a dysfunction of the respiratory muscles that is partly responsible for the dysarthria (Murdoch et al., 1989). Indeed there are, within overall poor control of expiratory airflow, an alteration of the air quantity needed for the vibration of vocal cords (Jiang et al., 1999a ; Solomon \& Hixon, 1993). However, the SGP is the result of a surge in air column by the pressure of lung with laryngeal resistance (Crevier-Buchman, 2007; Solomon, 2007). In the particular context of this study, when measuring IOP via the GSP, the glottis is open, at that time so it's a pressure gradient which is measured and not a static value. This gradient is the result of coordinated action between the respiratory muscles and laryngeal floor, so it indicates pneumophonic coordination quality. In PD, the fall in pulmonary pressure associated with hypokinetic movements of laryngeal muscles induced an alteration of the SGP. So we have shown in this study that it is possible to consider the GSP, or IOP, as a strong indicator of Parkinsonian dysarthria in general and its pneumophonic side particularly. We confirm in same time the results already published in a preliminary study (Sarr et al., 2009). Therefore, the measurement of IOP may allow together, comparing OFF DOPA patients and control subjects, assessment of the disease impact on speech disorders and contribution to evaluation of somes therapies such as L-dopa and subthalamic nucleus stimulation on parkinsonian dysarthria. As a reminder in our study (Sarr et al., 2009), STN stimulation improves IOP significantly in the initial part of the expiratory phase.

Regarding the mean oral airflow (MOAF), no difference was found between patients in OFF DOPA and control subjects at the first and last measurement point (P1 and P6). That means patients and control subjects would develop the same speed to start and finish the sentence «Papa ne m'a pas parlé de beau-papa ». Difference between the two groups was only noted during the course of sentence production. Indeed at other measurement points (P2 to P5), the curve of control subjects is well above that of patients in OFF DOPA, the difference between the two groups was significant $(p=0.001)$. It is also found that the curve of control subjects had a more stable pace with its roughly more linear shape (See figure 7). This could reflect a greater mastery of oral airflow by control subjects. In other words, the relatively greater irregularity of the curve of average values of MOAF in patients could reinforce the idea of a less good control of the MOAF. The reported decrease of MOAF could merely be a consequence of the fall in IOP. For example, assuming that laryngeal resistance is constant, the drop in IOP is necessarily associated with diminution in MOAF. However it seems exist in this study a large variability in laryngeal resistance in patients, as an overview was provided us in the morphological analysis of their value curves. This suggests a relatively fluctuating fall in MOAF which may also be related to tissue properties, configuration of the glottis and impedance of the vocal apparatus (Jiang \& Tao, 2007). It is reported more generally in extrapyramidal syndromes glottic and supraglottic disorders such as movement disorders. These disorders can obstruct completely or partially the upper airway to induce sometimes severe airflow decrease (Vincken et al., 1984). The MOAF decline during speech production of PD patients could also be explained by similar mechanisms, among others.

Finally for the laryngeal resistance (LR), Parkinson's disease could induce a greater variability of this parameter in patients compared to control subjects, as evidenced by the general morphology of control subjects and OFF DOPA patients' curves. In other words, control subjects would have more stable values of LR, which would mean that Parkinson's disease induces instability of laryngeal resistance. The values of standard deviations 
significantly larger in OFF DOPA patients than control subjects, again reflecting greater variability in the values of LR at all measurement points, seem to confirm this trend (See Figure 9). The study of LR values distribution histogram in the two groups seems to be in the same direction. Indeed, the histogram shows a fairly symmetrical distribution for control subjects where OFF DOPA patients have more skewed distributions, with thus a tendency to give most often higher LR values compared to control subjects (See Figure 10).

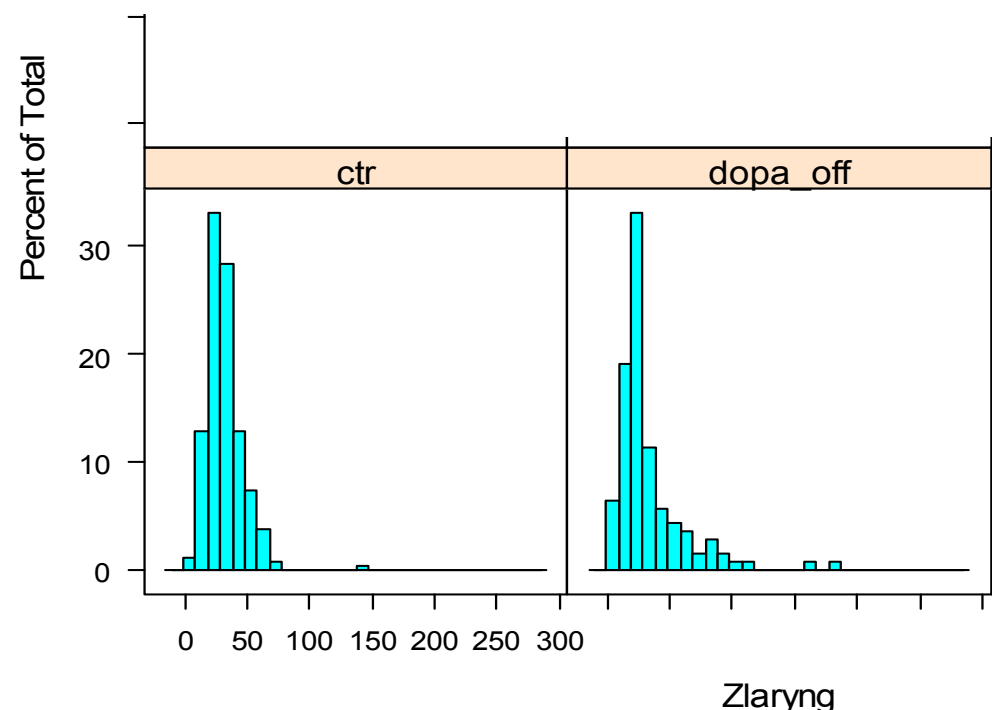

Fig. 10. Histogram of the distribution of values of laryngeal resistance (Zlaryng).

There is a fairly symmetrical distribution for control subjects, while values distributions are more skewed in OFF DOPA patients.

Laryngeal resistance is equal to the ratio of IOP on MOAF; its greater constancy among control subjects may indicate a more perfect mastery of these two parameters. Besides this relative constancy of laryngeal resistance in control subjects was found in the measures performed by Smitheran and Hixon (1981). Smitheran and Hixon measurements were performed to compare laryngeal resistance values in non-invasive technique of measurement with those of invasive procedures. The mean laryngeal resistance in their patients was $35.7+/-3.3 \mathrm{~cm}$ H20/LPS (all measurements are between 30 and 43, 1). Blosser et al. (1992) reported similar values with a mean of $38.4+/-7.43 \mathrm{~cm} \mathrm{H20/LPS.} \mathrm{In} \mathrm{addition}$ laryngeal resistance may reflect the larynx subject behavior. This has been demonstrated in a canine animal model which is able of maintaining, like humans, a constant subglottic pressure during phonation. In this model it was found a significant rise in laryngeal resistance when increasing the recurrent laryngeal nerve stimulation while the same nerve paralysis induced a significant drop of laryngeal resistance (Nasri et al., 1994). This significant rise in LR was also found in other disease involving larynx impairment with patients 'average to $65+/-8.15 \mathrm{~cm} \mathrm{H20/LPS} \mathrm{(Blosser} \mathrm{et} \mathrm{al.,} \mathrm{1992).} \mathrm{We} \mathrm{can} \mathrm{therefore} \mathrm{assume}$ that the instability of laryngeal resistance in OFF DOPA patients reflects a more variable behavior of their larynx, but also a greater fluctuation in IOP and MOAF. We know, as seen previously, that patients have IOP lower than those of control subjects at all measurement 
points. So the important rise of patients' laryngeal resistance in the first half of the sentence, beyond the intrinsic behavior of larynx, may result from a larger drop of their airflow as we had also seen. Therefore the decline in patients' IOP in the second half of the sentence would induce the consequent decline of their laryngeal resistance. That's why the global evolutionary pace of patients' curve shows increased laryngeal resistance in the first half of the sentence and significant drop in the second half. These high laryngeal resistances in the beginning of the sentence could be related to a lack of pneumophonic coordination, that is to say a kind of phase shift between the air expiratory thrust and resistance state of the larynx. Everything would go as if, when the expiratory air exerts its thrust, the larynx is still at resistance level higher than normal. The larynx would amount only to a resistance normal level later, which would explain the decrease of laryngeal resistance in the second half of the sentence. In short, this phenomenon simply imitate, but this time at the pneumophonic floor, the mechanism of control loss of voice onset time (VOT) which reflects a lack of coordination between the larynx and articulatory organs (Forrest et al. 1989; Lieberman et al., 1992).

It thus appears that there is in Parkinson's disease pneumophonic coordination impairments which are evidenced by the fall in IOP and that of MOAF in patients compared with control subjects. And it follows from the alteration of these two parameters a greater instability of laryngeal resistance which is none other than ratio of two above mentioned parameters. For didactic sake, we attempted to separately discuss the different parameters (IOP, MOAF and LR). However it should be borne in mind that these parameters are closely related functionally, and that any change in one inevitably has repercussions on the other two. Indeed, the SGP (reflected here by the IOP) depends on the air expiratory column thrust and laryngeal resistance (LR) while translaryngeal airflow (reflected here by the MOAF) is merely the result of the conflict between expiratory thrust forces (SGP) and laryngeal resistance (LR) forces (Crevier-Buchman, 2007; Solomon, 2007). Reported disturbances in the three parameters pose the problem of events' real chronology because of parameters' correlation. Is it the increase in LR at the beginning of the sentence which induces a fall in MOAF or, conversely, would it fall in MOAF resulting of expiratory thrust poor dynamic that would cause the increase in LR? It could probably be a simultaneous mechanism combining both alteration of expiratory dynamic (leading to fall in IOP and MOAF) and elevated laryngeal resistance notably at sentence beginning (reinforcing the fall in MOAF). Such a mechanism would both explain decrease in IOP and initial elevation of laryngeal resistance which both lead to a decline in MOAF that patients would be tempted to correct by vocal abuse. Finally, such a mechanism would fit perfectly to a lack of pneumophonic coordination imitating, as we noted above, the lack of coordination in phono-articulatory stage which induces the voice onset time (VOT).

\section{Conclusion}

Parkinson's disease, given the study of these three parameters, likely induces an alteration of pneumophonic coordination involving a decrease in IOP, a decrease in MOAF and instability of the LR. So the measurements of these three aerodynamics parameters, by reflecting the dysfunction induced by disease, may well be relevant factors in parkinsonian dysarthria evaluation. These parameters can also be valuable in evaluation of several therapies used in Parkinson's disease treatment in general and dysarthria in particular. A limit of the present work is the lack of acoustic parameters assessment. In fact we thought 
that the sentence "Papa ne m'a pas parlé de beau-papa" is less appropriate than other tasks such as sustained vowel for evaluation of acoustic parameters. In any case, increasingly, methods for assessing parkinsonian dysarthria should be larger, including both central and peripheral levels of speech production. Future research to better understand and assess parkinsonian dysarthria would benefit from taking more account of a more global study of dysarthria contours.

\section{References}

Abbs JH, Hartman DE, Vishwanat B. Orofacial motor control impairment in Parkinson's disease. Neurology 1987, 37: 394-398.

Ackermann H, Grone BF, Hoch G, Schonle PW. Speech freezing in Parkinson's disease: a kinematic analysis of orofacial movement by means of electromyographic articulography. Folia phoniat 1993, 45: 84-89.

Ackermann H, Ziegler W. Articulatory deficits in parkinsonian dysarthria: an acoustic analysis. Journal of Neurology, Neurosurgery, and Psychiatry 1991, 54: 1093-1098.

Baken RJ and Orlikoff RF (2000). Clinical Measurement of Speech and Voice, 2nd ed. (Singular, Thomson Learning, San Diego).

Barlow SM, Abbs JH. Force transducers for the evaluation of labial, lingual, and mandibular motor impairments. Journal of Speech and Hearing Research 1983, 26: 616-621.

Blosser S, Wigley FM, Wise RA. Increase in translaryngeal resistance during phonation in rheumatoid arthritis. Chest 1992, 102(2):387-90.

Caligiuri M. The influence of speaking rate on articulatory hypokinesia in parkinsonian dysarthria. Brain lang 1989, 36: 493-502.

Canter GJ. Speech characteristics of patients with Parkinson's disease: I Intensity, pitch and duration. J Speech Hearing Dis 1963, 28: 221-229.

Connor NP, Abbs JH. Task-dependent variations in parkinsonian motor impairment. Brain 1991, 114: 321-332.

Connor NP, Abbs JH, Cole KJ, Gracco VL. Parkinsonian deficits in serial multiarticulate movements for speech. Brain 1989, 112: 997-1009.

Crevier-Buchman L. Modélisation du fonctionnement laryngé. In Auzou P, Monnoury-Rolland V, Pinto S, Özsancak C (eds),Les dysarthries. Solal. Marseille. 2007 pp 91-100.

Darley FL, Aronson AE, Brown JR. Differential diagnostic patterns of dysarthria. Journal of Speech and Hearing Research 1969, 12: 246-269.

Darley FL, Aronson AE, Brown JR. (1975). Motor speech disorders, pp. 171-197. Saunders WB, Philadelphia.

Demolin D, Giovanni A, Hassid S, Heim C, Lecuit V, Socquet A (1997). "Direct and indirect measurements of subglottic pressure", Proc. Larynx 97, Marseille, p.69-72.

Duez D. Organisation temporelle de la parole et dysarthrie parkinsonienne. In Özsancak C, Auzou $\mathrm{P}$ (ed). Les troubles de la parole et de la déglutition dans la maladie de Parkinson. Solal. Marseille. 2005, pp 195-211.

Flint AJ, Black SE, Campbell-Taylor I, Gailey GF, Levinton C. Acoustic analysis in the differentiation of Parkinson's disease and major depression. Journal of Psycholinguistic Research 1992, 21: 383-389.

Forrest K, Weismer G, Turner GS. Kinematic, acoustic and perceptual analyses of connected speech produced by parkinsonian and normal geriatric adults. J Acoust Soc Am 1989, 85: 26082622. 
Fox C, Ramig LO. Vocal sound pressure level and self-perception of speech and voice in men and women with idiopathic Parkinson disease. American Journal of Speech-Language Pathology 1997, 6: 85-94.

Gentil M, Pollak P, Perret J. La dysarthrie parkinsonienne. Rev. Neurol. (Paris) 1995, 151, 2, 105-112.

Harel B, Cannizzaro M, Snyder PJ. Variability in fundamental frequency during speech in prodromal and incipient Parkinson's disease: a longitudinal case study. Brain Cognition 2004, 56: 24-29.

Hartelius L, Svensson P. Speech and swallowing symptoms associated with Parkinson's disease and Multiple sclerosis : A survey. Folia Phoniatr Logop 1994, 46: 9-17.

Hertrich I, Ackermann H. Acoustic analysis of speech prosody in Huntington's and Parkinson's disease: a preliminary report. Clin Ling Phonetics 1993, 7:285-297.

Ho AK, Iansek R, Bradshaw JL. Regulation of parkinsonian speech volume: the effect of interlocutor distance. J Neurol Neurosurg Psychiatry 1999, 67 :199-202.

Ho AK, Iansek R, Marigliani C, Bradshaw JL, Gates S. Speech impairment in a large sample of patients with Parkinson's disease. Behav Neurol 1998, 11: 131-137.

Hunker CJ, Abbs JH, Barlow SM. The relationship between parkinsonian rigidity and hypokinesia in the oro-facial system: a quantitative analysis. Neurology 1982, 32: 749-754.

Jankowski L, Purson A, Teston B, Viallet F. Effets de la L-Dopa sur la dysprosodie et le fonctionnement laryngien de patients parkinsoniens. Actes des XXVèmes journées d'études sur la parole, Fès, Maroc, 19-22 avril 2004.

Jiang J, Tao C. The minimum glottal airflow to initiate vocal fold oscillation. J Acoust Soc Am 2007, 121(5):2873-81.

Jiang J, Lin E, Wang J, Hanson DG. Glottographic measures before and after Levodopa treament in Parkinson's disease. Laryngoscope 1999, 109: 1287-1294.

Jiang J, O'Mara T, Chen HJ, Stern JI, Vlagos D, Hanson D. Aerodynamic measurements of patients with Parkinson's desease. J Voice 1999a, 13:583-591.

Jimenez-Jimenez FJ, Gamboa J, Nieto A, Guerrero J, Orti-Pareja M, Molina JA, Garcia-Albea E, Cobeta I. Acoustic voice analysis in untreated patients with Parkinson's disease. Parkinsonism \& Related Disorders 1997, 3: 111-116.

Kent RD, Rosenbek JC. Prosodic disturbance and neurologic lesions. Brain Lang 1982, 15: 259-291.

Leanderson R, Persson A, Ohman S. Electromyographic studies of facial muscle activity in speech. Acta Oto-laryngologica 1971, 72: 361-369.

Lieberman P, Kako E, Friedman J, Tajchman G, Feldman LS, Jiminez EB. Speech production, syntax comprehension and cognitive deficits in Parkinson's disease. Brain Lang 1992, 43: 169-189.

Logemann JA, Fisher HB, Boshes B, Blonsky ER. Frequency and cooccurrence of vocal tract dysfunctions in the speech of a large sample of Parkinson patients. J Speech Hear Dis 1978, 43: 47-57.

Ludlow CL, Connor NP, Bassich CJ. Speech timing in Parkinson's and Huntington's disease. Brain and Language 1987, 32: 195-214.

Ludlow CL, Bassich CJ. (1984). Relationship between perceptual ratings and acoustic measures of hypokinetic speech. In: McNeil M, Rosenbek JC, Aronson AE (eds), The dysarthrias : Physiology, acoustic, perception, management, pp. 163-196. College Hill Press, San Diego.

Metter EJ, Hanson WR. Clinical and acoustical variability in hypokinetic dysarthria. J Comm Disorders 1986, 19: 347-366. 
Monfrais-Pfauwadel MC. Palilalies et pseudobégaiements. In Özsancak C, Auzou P (eds). Les troubles de la parole et de la déglutition dans la maladie de Parkinson. Solal. Marseille. 2005, pp 213-222.

Murdoch BE, Chenery HJ, Bowler S, Ingram JC. Respiratory function in parkinson's subjects exhibiting a perceptible speech deficit: a kinematic and spirometric analysis. J Speech Hear Dis 1989, 54:610-626.

Nasri S, Namazie A, Kreiman J, Sercarz JA, Gerrat BR, Berke GS. A pressure-regulated model of normal and pathologic phonation. Otolaryngol Head Neck Surg. 1994, 111 (6):807-15.

Netsell R, Daniel B, Celesia GG. Acceleration and weakness in parkinsonian dysarthria. J Speech Hearing Dis 1975, 40: 170-178.

Robert D, Spezza C. La dysphonie parkinsonienne et les troubles articulatoires dans la dysarthrie parkinsonienne. In Özsancak C, Auzou P (eds). Les troubles de la parole et de la déglutition dans la maladie de Parkinson. Solal. Marseille. 2005, pp 131-159.

Sanabria J, Garcia Ruiz P, Guttierez R. The effect of Levodopa on vocal function in Parkinson's disease. Clin Neuropharmacol 2001, 24: 99-102.

Sarr MM, Pinto S, Jankowski L, Teston B, Purson A, Ghio A, Régis J, Peragut JC, Viallet F. Contribution de la mesure de la pression intra-orale pour la compréhension des troubles de la coordination pneumophonique dans la dysarthrie parkinsonienne. Rev Neurol 2009, 165: $1055-1061$.

Selby G. (1968). Parkinson's disease. In: PJ Winken \& GW Bruyn, Handbook of clinical neurology, pp. 173-211. North Holland Publishing Company, Amsterdam.

Smith ME, Ramig LO, Dromey C, Perez KS, Samandari R. Intensive voice treatment in Parkinson disease: laryngostroboscopic finding. Journal of Voice 1995, 9: 453-459.

Smitheran J., Hixon T. A Clinical method for estimating laryngeal airway resistance during vowel production. J Speech Hear Dis 1981, 46:138-146.

Solomon NP. La fonction respiratoire dans la production de parole. In Auzou P, Monnoury-Rolland V, Pinto S, Özsancak C (eds),Les dysarthries. Solal. Marseille. 2007 pp 44-55.

Solomon NP, Hixton TJ. Speech breathing in Parkinson's disease. Journal of Speech and Hearing Research 1993, 36: 294-310.

Teston B. L'étude instrumentale des gestes dans la production de la parole : Importance de l'aérophonométrie. In Auzou P, Monnoury-Rolland V, Pinto S, Özsancak C (eds),Les dysarthries. Solal. Marseille. 2007 pp 248-258.

Viallet F, Teston B. La dysarthrie dans la maladie de Parkinson. In Auzou P, Monnoury-Rolland V, Pinto S, Özsancak C (eds),Les dysarthries. Solal. Marseille. 2007 pp 375-382.

Viallet F, Teston B, Jankowski L, Purson A, Peragut J, Régis J, Witgas T. Effects of pharmacological versus electrophysical treatements on parkinsonian dysprosody. In: Speech prosody, pp 679-682. Aix-en-Provence (2002)

Vincken WG, Gauthier SG, Dollfuss RE, Hanson RE, Darauay CM, Cosio MG. Involvement of upper-airway muscles in extrapyramidal disorders. A cause of airflow limitation. N Engl J Med. 1984, 311(7):438-42.

Volkmann J, Hefter H, Lange HW, Freund HJ. Impairment of temporal organization of speech in basal ganglion diseases. Brain Lang 1992, 43: 386-399.

Weismer G. (1984) Articulatory characteristics of parkinsonian dysarthria. In: The dysarthrias: Physiology, acoustics, perception, management (McNeil M, Rosenbek J, Aronson AE, eds), pp 101-130. San Diego: College Hill Press.

Yuceturk AV, Yilmaz H, Egrilmez M, Karaca S. Voice analysis and videolaryngostroboscopy in patients with Parkinson's disease. Eur Arch Otorhinolaryngol 2002, 259: 290-293. 